\title{
Power to identify a genetic predictor of antihypertensive drug response using different methods to measure blood pressure response
}

\author{
Stephen T Turner ${ }^{1 *}$, Gary L Schwartz ${ }^{1}$, Arlene B Chapman ${ }^{3}$, Amber L Beitelshees ${ }^{4}$, John G Gums ${ }^{5,6}$, \\ Rhonda M Cooper-DeHoff ${ }^{5,7}$, Eric Boerwinkle ${ }^{8}$, Julie A Johnson ${ }^{5,7}$ and Kent R Bailey ${ }^{2}$
}

\begin{abstract}
Background: To determine whether office, home, ambulatory daytime and nighttime blood pressure (BP) responses to antihypertensive drug therapy measure the same signal and which method provides greatest power to identify genetic predictors of BP response.

Methods: We analyzed office, home, ambulatory daytime and nighttime BP responses in hypertensive adults randomized to atenolol $(N=242)$ or hydrochlorothiazide $(N=257)$ in the Pharmacogenomic Evaluation of Antihypertensive Responses Study. Since different measured BP responses may have different predictors, we tested the "same signal" model by using linear regression methods to determine whether known predictors of BP response depend on the method of BP measurement. We estimated signal-to-noise ratios and compared power to identify a genetic polymorphism predicting BP response measured by each method separately and by weighted averages of multiple methods.

Results: After adjustment for pretreatment BP level, known predictors of BP response including plasma renin activity, race, and sex were independent of the method of BP measurement. Signal-to-noise ratios were more than 2-fold greater for home and ambulatory daytime BP responses than for office and ambulatory nighttime BP responses and up to 11-fold greater for weighted averages of all four methods. Power to identify a genetic polymorphism predicting BP response was directly related to the signal-to-noise ratio and, therefore, greatest with the weighted averages.

Conclusion: Since different methods of measuring BP response to antihypertensive drug therapy measure the same signal, weighted averages of the BP responses measured by multiple methods minimize measurement error and optimize power to identify genetic predictors of BP response.
\end{abstract}

Keywords: hypertension, blood pressure monitoring, antihypertensive drug therapy, beta-blocker, thiazide diuretic, plasma renin activity

\section{Background}

Although office blood pressure (BP) measurements remain the standard-of-care, averages of out-of-office measurements are more reproducible [1]. Out-of-office averages have also been reported to be more strongly correlated with subclinical target organ damage $[2,3]$ and to better predict future cardiovascular disease

\footnotetext{
* Correspondence: sturner@mayo.edu

'Division of Nephrology and Hypertension, Department of Medicine, Mayo Clinic, Rochester, MN 55905, USA

Full list of author information is available at the end of the article
}

events [4-6] than office measurements. Not surprisingly, $\mathrm{BP}$ responses to antihypertensive drug therapy are more precisely and accurately determined by out-of-office than office measurements, which are influenced by white coat and placebo effects $[7,8]$. Consequently, greater use of out-of-office methods of BP measurement has been advocated for clinical decision-making and research [1].

More individualized approaches to antihypertensive drug therapy may become possible if genetic polymorphisms are discovered that improve the ability to

\section{() Biomed Central}


predict inter-individual differences in BP response [9]. Known predictors are limited to race, age, and plasma renin activity $[10,11]$, which explain less than $50 \%$ of interindividual variation in BP response to single-drug therapy $[12,13]$. Most previous studies have attempted to identify genetic or non-genetic predictors of office BP response, which is not very reproducible and correlates only modestly with home and ambulatory BP responses [7,8,14-16]. Whether out-of-office measurements of BP response can improve the ability to identify predictors of $\mathrm{BP}$ response has not been demonstrated. Method-specific measurement errors could account for differences in the magnitude of and correlation between office, home, ambulatory daytime and nighttime BP responses [8]. However, an additional possibility is that different $\mathrm{BP}$ response signals are measured by the different methods.

Since different BP response signals may have different predictors, our first objective in the present study was to test the "same signal" model by determining whether known predictors of $\mathrm{BP}$ response, i.e., race, age, and plasma renin activity, depend on the method of BP measurement. We analyzed data from the Pharmacogenomic Evaluation of Antihypertensive Responses (PEAR) study, in which BP responses to single-drug therapy with atenolol or hydrochlorothiazide were measured by all four methods $[7,8]$. In this context, our second objective was to estimate signal-to-noise ratios and compare the power to identify a genetic polymorphism predicting BP response when measured by each method separately and by weighted averages of multiple methods.

\section{Methods Participants}

The PEAR study [17]http://clinicaltrials.gov/ct2/show/ NCT00246519 was approved by the Institutional Review Board at each site, and all participants gave informed consent. At an initial consent and screening visit, trained study personnel administered standardized questionnaires, performed a limited physical examination, and obtained blood and urine samples for testing to establish eligibility for participation [11]. Participants were provided an automated sphygmomanometer (MicroLife 3 AC1-PC, Minneapolis MN), the adequacy of which has been previously validated [18], and withdrawn from previous antihypertensive drug therapy. The device was set to measure BP in triplicate with each activation and to store the average systolic and diastolic BPs and the time of each set of measurements. Participants were instructed to take readings daily in the seated position, one set of three readings in the morning upon arising from bed and a second set in the evening just before retiring. At subsequent study visits (prior to randomization and at the end of therapy), an additional set of three readings was obtained seated ( $>5$ minutes) in the office using the home monitor. In addition, 24hour ambulatory BP recordings were obtained at these visits using Spacelabs (Redmond WA) ambulatory monitors, model 90207, the adequacy of which has been previously validated [19]. Participants were instructed to conduct their usual daily activities while wearing the monitor, which was set to record BP four times per hour during the day (6 AM to $10 \mathrm{PM}$ ) and twice per hour during the night (10 PM to $6 \mathrm{AM})$. The average ( \pm standard deviation) number of ambulatory measurements was $67 \pm 10$ during daytime hours and $15 \pm 3$ during nighttime hours.

At the end of the drug-free washout period, fasting blood samples were drawn in the seated position after ambulation for measurement of plasma renin activity [11]. To qualify for randomization, the average home diastolic BP in the previous week had to be $\geq 85 \mathrm{mmHg}$ (consisting of at least five morning and five evening sets of readings) and the average office diastolic BP $\geq 90$ $\mathrm{mmHg}$. Participants received either atenolol or hydrochlorothiazide, starting at $50 \mathrm{mg}$ or $12.5 \mathrm{mg}$ daily, respectively, for two weeks, after which, if BP remained $>120 / 70 \mathrm{mmHg}$, the doses were increased to $100 \mathrm{mg}$ or $25 \mathrm{mg}$ daily, respectively, for six additional weeks.

\section{Statistical analysis}

Analyses were performed with Statistical Analysis System software, version 9.1 (SAS, Raleigh-Durham NC). Statistical significance was defined a priori by $P<0.05$. The BP response to each drug was calculated for each measurement method by subtracting the pretreatment average from the post-treatment average. The home BP averages consisted of at least five of seven morning and evening sets of three readings taken during the week prior to the pre- and post-treatment study visits (i.e., at least 30 and up to 42 measurements prior to each visit). Multiple-variable linear regression analyses were performed to identify participant characteristics that made additive, statistically independent contributions to the prediction of systolic and diastolic BP response to each drug. In preliminary analyses, we found that higher pretreatment BP level was associated with greater BP response, as expected [20]. Because we sought to evaluate predictors that are independent of the pretreatment BP level, we first regressed out the effects of pretreatment BP level and then modeled the effects of other known predictors of BP response $[10,11]$ as well as other variables measured at the consent and screening visit $[11,17]$. Final multiple-variable models were derived using a backward stepwise elimination procedure, retaining only the predictors of both systolic and diastolic BP responses to either drug. In the initial models that included race and pretreatment plasma renin 
activity, age was not a statistically significant predictor of $\mathrm{BP}$ responses and was not retained in the final models.

To determine whether known predictors of $\mathrm{BP}$ response depend on the method of measuring $\mathrm{BP}$ response, we compared models in which regression coefficients were constrained to be identical among measurement methods or allowed to differ among methods by including interactions of each predictor with the method of BP measurement. Considering the model in which the regression coefficients were identical across methods as a null hypothesis, we attempted to detect any departures indicating dependency of the predictors on method of BP measurement that would lead us to reject the "same signal" model. This analysis used PROC GENMOD in SAS, which adjusts for the correlation among the four BP response measurements within each participant.

To estimate signal-to-noise ratios, the covariance matrix of the four measured BP responses was used to estimate the signal and noise components for each method of measuring BP response after regressing out the method-specific effects of pretreatment BP level. The correlation coefficient between BP responses measured by two methods provides a dimensionless measure of how much the two responses covary (change together); the covariance between them expresses the correlation in units of the two BP responses multiplied together $\left(\mathrm{mm}^{2} \mathrm{Hg}\right)$ and is the variance shared between them, i.e., the signal variance. Since each pair-wise covariance provides an unbiased estimate of the signal variance, we used the average of the six pairwise covariances as the BP response signal. Subtracting the signal from the method-specific total variance provided an estimate of the method-specific error variance or noise.

We examined implications of the signal-to-noise analyses for accomplishing the goal of the PEAR and other pharmacogenomic studies. Specifically, we compared power and samples sizes required to identify a genetic polymorphism that predicts $\mathrm{BP}$ response when measured by each method separately and by weighted averages of the responses measured by multiple methods. The rationale for the weighted averages was to increase the signalto-noise ratio (and power) by minimizing the error variance. Two different combinations of the measured BP responses were considered: a weighted average of all four methods and a weighted average of the office and home $\mathrm{BP}$ responses. The weighted average combinations were determined based on the row sums of the inverse of the inter-method covariance matrices, which provide weights that minimize the variance [21].

For the power and samples size calculations, we assumed that a genetic polymorphism with a minor allele frequency of 0.2 influences the BP response signal with an effect size that can be detected with $80 \%$ power in a sample of $\mathrm{N}=300$ at a genome-wide significance level of $5 \times 10^{-8}$. This $P$-value was originally suggested for genome-wide association analysis of 1 million single nucleotide polymorphisms using a Bonferroni correction for multiple testing [22]. Based on the estimated signal variances, we calculated the allele effect sizes (in $\mathrm{mmHg} /$ allele) and the percentage of variation in the BP response $\left(R^{2} \times 100 \%\right)$ explained by the polymorphism. We then calculated the power to detect the polymorphism in a sample of $\mathrm{N}=300$ when the $\mathrm{BP}$ response is measured by each method separately and weighted averages of multiple methods, and the corresponding sample sizes required to maintain $80 \%$ power.

\section{Results \\ Sample description}

Five hundred and ninety-five study participants had complete measurements of office, home, and ambulatory daytime and nighttime BP responses (Table 1). Of these, 293 participants were randomized to atenolol (49\%) and 302 to hydrochlorothiazide (51\%) treatment. Mean values and relative frequencies of participant characteristics measured prior to randomization did not differ significantly between the atenolol and hydrochlorothiazidetreated groups [11] (not shown).

\section{Office, home, and ambulatory BP response}

Means and standard deviations of the systolic and diastolic BP responses differed among measurement methods (Table 2). For systolic BP response, office measurements had the greatest mean declines and home measurements the smallest mean declines in response to each drug (Table 2). For diastolic BP response, office measurements also had the greatest mean declines in response to each drug; ambulatory nighttime measurements had the smallest mean decline in response to atenolol and home measurements the smallest mean decline in response to hydrochlorothiazide. Correlation coefficients between the office, home, ambulatory daytime and nighttime BP responses were modest in magnitude (not shown), ranging from 0.36 to 0.71 after adjustment for differences in pretreatment BP levels (all $P<0.0001)$.

\section{The BP response signal and its predictors}

We assessed whether the office, home, and ambulatory daytime and nighttime BP responses measure the same $\mathrm{BP}$ response signal by determining whether the predictors of BP response depend on the method of BP measurement (see Methods). After adjustment for pretreatment BP level, none of the predictors of BP response depended upon of the method of BP 
Table 1 Descriptive characteristics of study participants

\begin{tabular}{|c|c|}
\hline & Mean \pm standard deviation or $\mathrm{N}(\%)$ \\
\hline$\overline{N(\%)}$ & $595(100)$ \\
\hline Randomized to hydrochlorothiazide, N (\%) & $302(51)$ \\
\hline Age, years & $49.3 \pm 9.1$ \\
\hline Male, N (\%) & $280(47)$ \\
\hline Black, N (\%) & $245(41)$ \\
\hline $\mathrm{BMI}, \mathrm{kg} \cdot \mathrm{m}^{-2}$ & $30.6 \pm 5.6$ \\
\hline Hypertension duration, years & $7.1 \pm 7.2$ \\
\hline Antihypertensive medication, N (\%) & $492(91)$ \\
\hline Current smoker, $\mathrm{N}(\%)$ & $69(13)$ \\
\hline Glucose, $\mathrm{mg} \cdot \mathrm{dL}^{-1}$ & $94.8 \pm 10.5$ \\
\hline Creatinine, $\mathrm{mg} \cdot \mathrm{dL}^{-1}$ & $0.9 \pm 0.2$ \\
\hline Serum ALT, $U \cdot L^{-1}$ & $29.1 \pm 15.9$ \\
\hline Plasma renin activity, $\mathrm{ng} \cdot \mathrm{mL}^{-1} \cdot \mathrm{hr}^{-1}$ & $1.0 \pm 1.2$ \\
\hline Resting heart rate, beat $\cdot \mathrm{min}^{-1}$ & $71.0 \pm 10.2$ \\
\hline Screening office systolic BP, mmHg & $137.9 \pm 13.8$ \\
\hline Screening office diastolic BP, $\mathrm{mmHg}$ & $89.5 \pm 8.8$ \\
\hline Pretreatment systolic blood pressure, $\mathrm{mmHg}$ & $151.5 \pm 13.8$ \\
\hline Pretreatment diastolic blood pressure, $\mathrm{mmHg}$ & $98.2 \pm 6.3$ \\
\hline
\end{tabular}

BMI, body mass index; ALT, alanine aminotransferase; BP, mmHg. Characteristics were measured at the screening visit except pretreatment systolic and diastolic BP and plasma renin activity were measured at the end of the drug-free washout period prior to initiating atenolol or hydrochlorothiazide therapy.

measurement (analyses not shown). For all four methods of measuring BP response, the identified predictors included race, plasma renin activity, and sex (Table 3). As expected, black race was associated with lesser systolic and diastolic BP responses to atenolol and greater responses to hydrochlorothiazide; and greater log renin was associated with greater systolic and diastolic BP responses to atenolol and lesser responses to hydrochlorothiazide [11]. Male sex was independently associated with lesser systolic and diastolic BP responses to each drug. Greater log hypertension years and greater serum ALT were each independently associated with greater systolic and diastolic BP responses to atenolol but not to hydrochlorothiazide.

\section{Signal-to noise-ratios}

Inferring that all four methods measure the same BP response signal, we estimated the signal variance (see Methods) and calculated the method-specific error variance (noise) and signal-to-noise ratio for each measured $\mathrm{BP}$ response (Figures 1 and 2). The home and ambulatory daytime BP responses had the largest signal-to-noise ratios and the ambulatory nighttime and office BP responses the smallest signal-to-noise ratios (Figure 2). The signal-tonoise ratios of the home and ambulatory daytime BP responses were similar in magnitude and up to 4-fold greater than the signal-to-noise ratios of the office and ambulatory nighttime BP responses, which were mostly less than 1 (more noise than signal). Weighted averages of

Table 2 Blood Pressure Responses to Monotherapy by Measurement Method

\begin{tabular}{|c|c|c|c|}
\hline & All $N=595$ & Atenolol N = 293 & Hydrochlorothiazide $\mathrm{N}=302$ \\
\hline \multicolumn{4}{|l|}{ Systolic BP Response, mmHg } \\
\hline Office & $-13.4 \pm 14.7$ & $-13.5 \pm 15.6$ & $-13.2 \pm 13.7$ \\
\hline Home & $-8.8 \pm 9.8$ & $-8.3 \pm 10.4$ & $-9.4 \pm 9.1$ \\
\hline Ambulatory daytime & $-11.5 \pm 10.5$ & $-12.2 \pm 11.1$ & $-10.8 \pm 9.8$ \\
\hline Ambulatory nighttime & $-9.7 \pm 12.5$ & $-8.9 \pm 12.9$ & $-10.6 \pm 12.1$ \\
\hline Contrast $P$ value & $<0.001$ & $<0.001$ & $<0.001$ \\
\hline \multicolumn{4}{|l|}{ Diastolic BP Response, mmHg } \\
\hline Office & $-8.6 \pm 8.9$ & $-10.5 \pm 9.4$ & $-6.8 \pm 7.9$ \\
\hline Home & $-6.6 \pm 6.5$ & $-7.8 \pm 6.8$ & $-5.3 \pm 6.0$ \\
\hline Ambulatory daytime & $-7.6 \pm 7.6$ & $-9.2 \pm 7.9$ & $-6.1 \pm 7.0$ \\
\hline Ambulatory nighttime & $-6.8 \pm 9.5$ & $-7.0 \pm 10.0$ & $-6.5 \pm 9.0$ \\
\hline Contrast $P$ value & $<0.001$ & $<0.001$ & 0.10 \\
\hline
\end{tabular}

BP, blood pressure. 
Table 3 Multi-variable linear regression modeling of predictors of blood pressure response signal after adjustment for pretreatment blood pressure level

\begin{tabular}{|c|c|c|c|c|}
\hline & \multicolumn{2}{|c|}{ BP Response to Atenolol $(\mathrm{N}=293)$} & \multicolumn{2}{|c|}{ BP Response to Hydrochlorothiazide $(\mathrm{N}=302)$} \\
\hline & Systolic $\beta \pm \mathrm{SE}$ & Diastolic $\beta \pm S E$ & Systolic $\beta \pm S E$ & Diastolic $\beta \pm \mathrm{SE}$ \\
\hline Intercept & $-14.1 \pm 0.9 \S$ & $-11.8 \pm 0.6 \S$ & $-11.8 \pm 0.7 \S$ & $-6.7 \pm 0.5 \S$ \\
\hline Race: Black & $6.3 \pm 1.1 \S$ & $4.8 \pm 0.8 \S$ & $-2.9 \pm 0.9 \neq$ & $-2.0 \pm 0.6 \neq$ \\
\hline Log Plasma Renin Activity & $-4.4 \pm 0.5 \S$ & $-2.5 \pm 0.3 \S$ & $0.7 \pm 0.5$ & $0.7 \pm 0.3^{*}$ \\
\hline Sex: Male & $2.3 \pm 1.0^{*}$ & $2.9 \pm 0.7 \S$ & $4.2 \pm 0.8 \S$ & $2.7 \pm 0.5 \S$ \\
\hline Log Hypertension Years & $1.3 \pm 0.5^{*}$ & $1.0 \pm 0.4 \dagger$ & - & - \\
\hline Serum ALT & $0.08 \pm 0.03 \dagger$ & $0.04 \pm 0.02^{*}$ & - & - \\
\hline Model $R^{2} \times 100 \%$ & $22 \%$ & $23 \%$ & $11 \%$ & $16 \%$ \\
\hline
\end{tabular}

$\mathrm{BP}$, blood pressure; $\beta$, regression coefficient; $\mathrm{SE}$, standard error. Model parameters are estimated at the mean values for each quantitative predictor variable in a combined dataset used to model the predictors of office, home, ambulatory daytime and nighttime BP responses after adjustment for pretreatment BP levels. $P$ values for tests of model parameters $=0:{ }^{*}, \leq 0.05 ; \dagger, \leq 0.01 ; \neq, \leq 0.001 ; \S, \leq 0.0001 . R^{2} \times 100 \%$ is the percentage of variation in the office BP response explained by the model predictors.

all four measured BP responses improved the signal-tonoise ratios up to 4-fold compared to the home BP responses and up to 19 -fold compared to the office $\mathrm{BP}$ responses. Weighted averages of the home and office BP responses improved the signal-to-noise ratios modestly compared to the home BP responses (by $24 \%$ at most). The weightings, provided in Additional file 1: Table S1, minimized the error variance (noise) of the average BP responses (see Methods), thereby accounting for the improvement in signal-to-noise ratios.

Power and sample size needed to identify a genetic predictor of blood pressure response

We assumed that a genetic polymorphism with minor allele frequency of 0.2 influences the BP response signal and is detected with $80 \%$ power at genome-wide significance level of $5 \times 10^{-8}$ (see Methods) [22]. The T-statistic for association of the polymorphism with BP response is $6.29 ; 11.6 \%$ of the signal variation is explained $\left(R^{2} \times 100 \%\right)$; and the corresponding effect sizes ( $\beta$-coefficients) in $\mathrm{mm} \mathrm{Hg}$ per allele are 5.22/3.36 for the systolic/diastolic BP responses to atenolol and 4.04/ 2.63 for the systolic/diastolic BP responses to hydrochlorothiazide. Based on the signal and noise analyses, power to detect this polymorphism in a sample size of $\mathrm{N}=300$ declined for all methods of measuring BP response when compared to a perfect method capable of measuring only signal and no noise (Table 4). Power declined most markedly for the office BP responses (to $<5 \%$ power) and was only maintained at $>50 \%$ for the
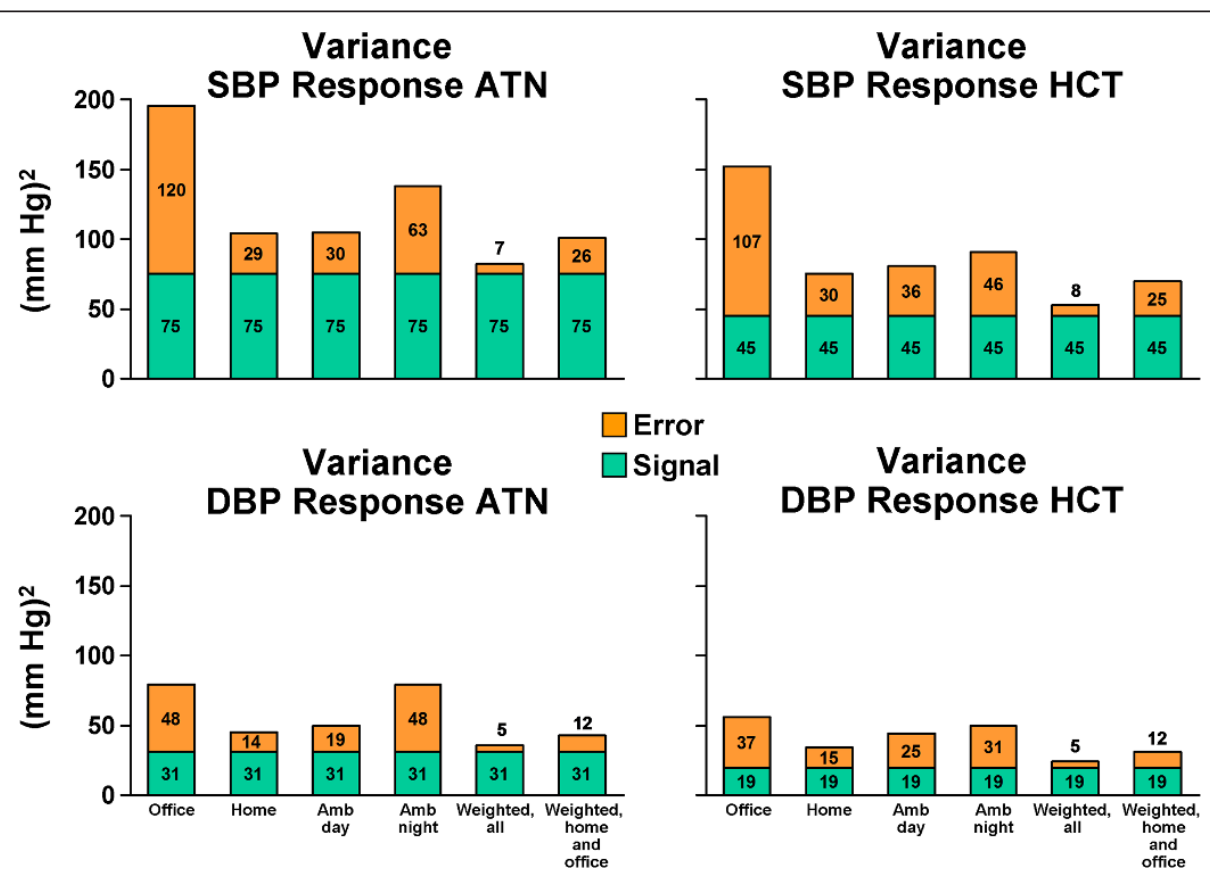

Error

Signal

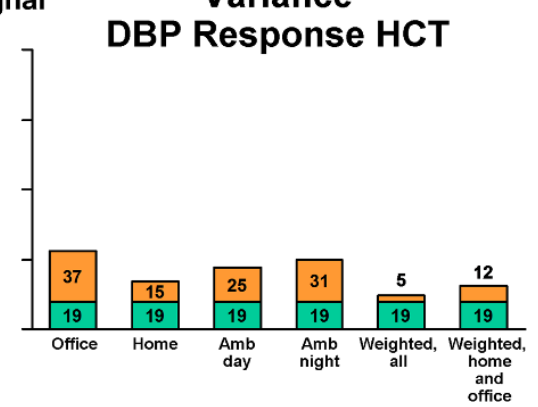

Figure 1 Signal, noise, and total variances of the measured blood pressure responses to single-drug therapy with atenolol or hydrochlorothiazide and their weighted averages. 


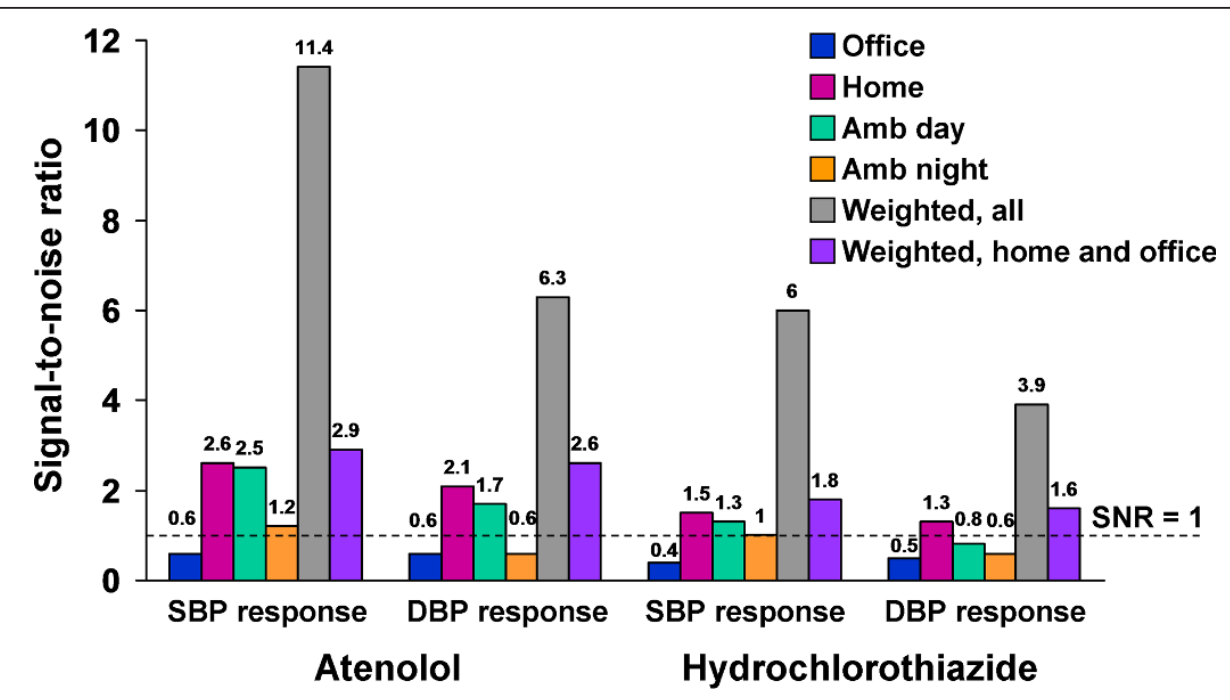

Figure 2 Signal-to-noise ratios of the measured blood pressure responses to single-drug therapy with atenolol or hydrochlorothiazide and their weighted averages.

weighted averages of all four methods. Alternatively, to maintain $80 \%$ power the sample sizes would need to be increased for all methods of measuring BP response when compared to the $\mathrm{N}=300$ sample size for the perfect measurement of signal without noise (Table 4). The sample sizes increased most markedly for the office BP responses (by $>200 \%$ ), but only modestly for the weighted averages of all four methods (by $\leq 26 \%$ ).

\section{Discussion}

Our first objective was to assess whether office, home, and ambulatory daytime and nighttime measurements of

Table 4 Power and sample sizes to detect single nucleotide polymorphism influencing BP response measured by office, home, ambulatory daytime and nighttime blood pressure

\begin{tabular}{|c|c|c|c|c|}
\hline & \multicolumn{4}{|c|}{ Drug } \\
\hline & \multicolumn{2}{|c|}{ Atenolol } & \multicolumn{2}{|c|}{ Hydrochlorothiazide } \\
\hline & Power, $\mathrm{N}=300$ & $\mathrm{~N}, 80 \%$ power & Power, $\mathrm{N}=300$ & $\mathrm{~N}, 80 \%$ power \\
\hline Systolic BP response signal & $80 \%$ & 300 & $80 \%$ & 300 \\
\hline \multicolumn{5}{|l|}{ Measurement methods } \\
\hline Office & $4 \%$ & 780 & $1.5 \%$ & 1013 \\
\hline Home & $42 \%$ & 416 & $24 \%$ & 500 \\
\hline Ambulatory day & $41 \%$ & 420 & $18 \%$ & 540 \\
\hline Ambulatory night & $17 \%$ & 552 & $12 \%$ & 607 \\
\hline \multicolumn{5}{|l|}{ Weighted averages } \\
\hline All methods & $70 \%$ & 328 & $61 \%$ & 353 \\
\hline Home and office & $45 \%$ & 404 & $30 \%$ & 467 \\
\hline Diastolic BP response signal & $80 \%$ & 300 & $80 \%$ & 300 \\
\hline \multicolumn{5}{|l|}{ Measurement methods } \\
\hline Office & $5 \%$ & 764 & $3 \%$ & 884 \\
\hline Home & $39 \%$ & 426 & $19 \%$ & 537 \\
\hline Ambulatory day & $39 \%$ & 426 & $19 \%$ & 537 \\
\hline Ambulatory night & $27 \%$ & 484 & $7 \%$ & 695 \\
\hline \multicolumn{5}{|l|}{ Weighted averages } \\
\hline All methods & $63 \%$ & 348 & & 379 \\
\hline Home and office & $42 \%$ & 416 & $26 \%$ & 489 \\
\hline
\end{tabular}

$\mathrm{BP}$, blood pressure. The power and sample size estimates are for a single nucleotide polymorphism with minor allele frequency of 0.2 that influences the BP response signal with an effect size detected with $80 \%$ power in a sample of $\mathrm{N}=300$ at a genome-wide significance level of $5 \times 10^{-8}$ (see Methods). The estimates assume that the BP response signal can be measured without error. 
$\mathrm{BP}$ response to single-drug therapy measure the same $B P$ response signal. Since different BP response signals may have different predictors, this assessment was based on determining whether known predictors of $\mathrm{BP}$ response [11] depend on the method of BP measurement. After adjustment for the method-specific effects of pretreatment BP level, the identified predictors of BP response were independent of the method of BP measurement. This finding supports the inference that office, home, and ambulatory daytime and nighttime BP responses measure the same BP response signal.

Our second objective was to estimate signal-to-noise ratios and compare the power to identify a genetic polymorphism predicting BP response when measured by each method separately and by weighted averages of multiple methods. Estimation of the BP response signal allowed us to also compare each method with a theoretically perfect measurement consisting of pure signal and no noise. We reasoned that greater signal-to-noise ratios would translate into greater power and smaller sample sizes required to identify a polymorphism influencing BP response to antihypertensive drug therapy. Not surprisingly, signal-to-noise ratios were greater for the home and ambulatory daytime methods, which are based on more measurements per subject and have smaller error variances, than for the office and ambulatory nighttime methods, which are based on fewer measurements per subject and have larger error variances. Particularly unsettling were the signal-to-noise ratios less than one for office and ambulatory nighttime BP responses, indicating more noise than signal for these methods. Such measurement imprecision could account for limited success in previous studies to identify predictors of office BP response [12,23] and the requirement for sample sizes in the tens of thousands for genomewide association analyses of BP level [24]. Moreover, the profound lack of power to identify a genetic predictor of $\mathrm{BP}$ response in sample sizes $\leq 300$, and the large increases in sample size required to maintain $80 \%$ power, emphasizes the need for more precise methods of measuring BP response than office BP measurements provide [25].

Although the home and ambulatory daytime BP responses provided greater power than the office and ambulatory nighttime BP responses, the estimated sample size required to maintain $80 \%$ power with either method was still in excess of the number of participants randomized to each single-drug therapy in the PEAR study (i.e., $\mathrm{N}=400$ ). Consequently, we pursued additional strategies to increase power by combining all of the measurements from multiple methods in a weighted average, with the weights chosen to minimize the error variance (noise) and maximize the signal-to-noise ratio of the resulting average. We provided two examples: a weighted average of measurements from all four methods and a weighted average of the home and office measurements. The latter uses the two most feasible and widely available methods of measuring antihypertensive drug responses. While both weighted averages demonstrated improvements in the signal-to-noise ratios relative to the separate methods of measuring BP response, only with the weighted average of all four methods was power maintained at $80 \%$ without an increase in sample size exceeding the $N=400$ randomized to each singledrug therapy in the PEAR study. These signal and noise analyses, power calculations, and sample size estimates based on the PEAR study emphasize the "make-orbreak" contribution that precision in measurement of the phenotype can make to success of genome-wide association studies [26].

Given our interest in the PEAR study to identify new predictors of BP response, several additional results of our analyses are noteworthy. First, although signal variances were greater for the systolic than the diastolic BP responses, the error variances were also greater and the signal-to-noise ratios differed little between the systolic and diastolic BP responses. This finding suggests that neither phenotype affords greater opportunity than the other to identify its predictors. This suggestion is supported by the finding that each known predictor was a statistically significant predictor of both systolic and diastolic BP responses (Table 3). Second, greater signal and signal-to-noise ratios for the $\mathrm{BP}$ responses to atenolol might suggest greater predictability of BP response to atenolol than to hydrochlorothiazide. This suggestion is supported by the finding that two identified predictors of $\mathrm{BP}$ response to atenolol were not predictors of $\mathrm{BP}$ response to hydrochlorothiazide (Table 3). Third, male sex was associated with lesser systolic and diastolic BP responses to both atenolol and hydrochlorothiazide in this study and to hydrochlorothiazide in a previous pharmacogenetic study [12]. Male sex was also previously associated with lesser responses to quinapril [27] and candesartan [13]. To our knowledge, this apparently consistent association of male sex with lesser BP response to drugs from different pharmacological classes has not been previously recognized.

Despite many studies of antihypertensive drugs conducted since the 1950s, few patient characteristics have been identified that predict inter-individual differences in BP responses. Methods that reduce the error in measuring blood pressure response, especially weighted averages of the responses measured by multiple methods, improve signal-to-noise ratios and provide greater power to identify the predictors of response in smaller sample sizes. Their incorporation in the design of pharmacogenomic studies such as the PEAR study will be critical to success in identifying novel genetic 
polymorphisms that improve the ability to predict $\mathrm{BP}$ response to antihypertensive drug therapy.

\section{Conclusion}

Since different methods of measuring BP response to antihypertensive drug therapy measure the same signal, weighted averages of the BP responses measured by multiple methods minimize measurement error and optimize power to identify genetic predictors of BP response.

\section{Additional material}

Additional file 1: Additional file 1: Table S1. Esimated weights for

calculation of minimum variance weighted average blood pressure responses.

\section{Acknowledgements}

We gratefully acknowledge the valuable contributions of the study participants, support staff, and study physicians: Drs. George Baramidze, R. Whit Curry, Karen Hall, Karen Hall, Frederic Rabari-Oskoui, Dan Rubin, and Siegfried Schmidt. In addition, we gratefully acknowledge the biostatistical analyses of Daniel Crusan. Funding was from NIH Pharmacogenetics Research Network grant U01-GM074492; K23 grants HL091120 (A. L. Beitelshees) and HL086558 (R. M. Cooper-DeHoff); CTSA grants UL1RR092890 (University of Florida), UL1-RR025008 (Emory University), and UL1RR024150 (Mayo Clinic); and funds from the Mayo Foundation.

\section{Author details}

'Division of Nephrology and Hypertension, Department of Medicine, Mayo Clinic, Rochester, MN 55905, USA. Division of Biostatistics, Department of Health Sciences Research, Mayo Clinic, 200 First Street S.W, Rochester, MN, USA. ${ }^{3}$ Renal Division, Department of Medicine, Emory University School of Medicine, Atlanta, Georgia, USA. ${ }^{4}$ Department of Medicine, University of Maryland School of Medicine, Baltimore, MD, USA. ${ }^{5}$ Department of Pharmacotherapy and Translational Research and Center for Pharmacogenomics, University of Florida, Gainesville, FL, USA. ${ }^{6}$ Department of Community Health and Family Medicine, University of Florida, Gainesville, FL, USA. ${ }^{7}$ Department of Medicine, University of Florida, Gainesville, FL, USA. ${ }^{8}$ Human Genetics and Institute of Molecular Medicine, University of Texas Health Science Center, Houston, TX, USA.

\section{Authors' contributions}

ST and GS participated in the design of the study, collected and analyzed the data, and drafted and revised the manuscript. AB, JG, RCD participated in the design of the study, collected the data and participated in revision of the manuscript. JJ conceived and designed the study, collected the data, and participated in revision of the manuscript. EB participated in design of the study and revision of the manuscript. KB participated in design of the study, analyzed the data, and participated in drafting and revision of the manuscript. All authors read and approved the final manuscript

\section{Competing interests}

The authors declare that they have no competing interests.

Received: 20 December 2011 Accepted: 13 March 2012 Published: 13 March 2012

\section{References}

1. Parati G, Omboni S, Bilo G: Why Is Out-of-Office Blood Pressure Measurement Needed? Home Blood Pressure Measurements Will Increasingly Replace Ambulatory Blood Pressure Monitoring in the Diagnosis and Management of Hypertension. Hypertension 2009, 54:181-187.
2. Appel LJ, Stason WB: Ambulatory blood pressure monitoring and blood pressure self-measurement in the diagnosis and management of hypertension. Ann Intern Med 1993, 118:867-882.

3. Mancia G, Parati G: Ambulatory blood pressure monitoring and organ damage. Hypertension 2000, 36:894-900.

4. Bobrie G, Chatellier G, Genes N, Clerson P, Vaur L, Vaisse B, Menard J, Mallion JM: Cardiovascular prognosis of "masked hypertension" detected by blood pressure self-measurement in elderly treated hypertensive patients. JAMA 2004, 291:1342-1349.

5. Staessen JA, Den Hond E, Celis H, Fagard R, Keary L, Vandenhoven G, O'Brien ET: Antihypertensive treatment based on blood pressure measurement at home or in the physician's office: a randomized controlled trial. JAMA 2004, 291:955-964.

6. Staessen JA, Thijs L, Fagard R, O'Brien ET, Clement D, de Leeuw PW Mancia G, Nachev C, Palatini P, Parati G, et al: Predicting cardiovascular risk using conventional vs ambulatory blood pressure in older patients with systolic hypertension. Systolic Hypertension in Europe Trial Investigators [see comments]. JAMA 1999, 282:539-546.

7. Mancia G, Parati G: Office compared with ambulatory blood pressure in assessing response to antihypertensive treatment: a meta-analysis. J Hypertens 2004, 22:435-445.

8. Ishikawa J, Carroll DJ, Kuruvilla S, Schwartz JE, Pickering TG: Changes in home versus clinic blood pressure with antihypertensive treatments: a meta-analysis. Hypertension 2008, 52:856-864.

9. Turner ST, Schwartz GL, Boerwinkle E: Personalized medicine for high blood pressure. Hypertension 2007, 50:1-5.

10. Preston RA, Materson BJ, Reda DJ, Williams DW, Hamburger RJ, Cushman WC, Anderson RJ: Age-race subgroup compared with renin profile as predictors of blood pressure response to antihypertensive therapy. Department of Veterans Affairs Cooperative Study Group on Antihypertensive Agents. JAMA 1998, 280:1168-1172.

11. Turner ST, Schwartz GL, Chapman AB, Beitelshees AL, Gums JG, CooperDeHoff RM, Boerwinkle E, Johnson JA, Bailey KR: Plasma renin activity predicts blood pressure responses to beta-blocker and thiazide diuretic as monotherapy and add-on therapy for hypertension. Am J Hypertens 2010, 23:1014-1022.

12. Chapman AB, Schwartz GL, Boerwinkle E, Turner ST: Predictors of antihypertensive response to a standard dose of hydrochlorothiazide for essential hypertension. Kidney Int 2002, 61:1047-1055.

13. Canzanello VJ, Baranco-Pryor E, Rahbari-Oskoui F, Schwartz GL, Boerwinkle E, Turner ST, Chapman AB: Predictors of blood pressure response to the angiotensin receptor blocker candesartan in essential hypertension. Am J Hypertens 2008, 21:61-66.

14. Finkielman JD, Schwartz GL, Chapman AB, Boerwinkle E, Turner ST: Reproducibility of blood pressure response to hydrochlorothiazide. I Clin Hypertens (Greenwich) 2002, 4:408-412.

15. Finkielman JD, Schwartz GL, Chapman AB, Boerwinkle E, Turner ST: Lack of agreement between office and ambulatory blood pressure responses to hydrochlorothiazide. Am J Hypertens 2005, 18:398-402.

16. Beitelshees AL, Gong Y, Bailey KR, Turner ST, Chapman AB, Schwartz GL, Gums JG, Boerwinkle E, Johnson JA: Comparison of office, ambulatory, and home blood pressure antihypertensive response to atenolol and hydrochlorthiazide. J Clin Hypertens (Greenwich) 2010, 12:14-21.

17. Johnson JA, Boerwinkle E, Zineh I, Chapman AB, Bailey K, CooperDeHoff RM, Gums J, Curry RW, Gong Y, Beitelshees AL, et al: Pharmacogenomics of antihypertensive drugs: rationale and design of the Pharmacogenomic Evaluation of Antihypertensive Responses (PEAR) study. Am Heart J 2009, 157:442-449.

18. Topouchian JA, El Assaad MA, Orobinskaia LV, El Feghali RN, Asmar RG: Validation of two devices for self-measurement of brachial blood pressure according to the International Protocol of the European Society of Hypertension: the SEINEX SE-9400 and the Microlife BP 3AC1-1. Blood Press Monit 2005, 10:325-331.

19. O'Brien E, Mee F, Atkins N, O'Malley K: Accuracy of the SpaceLabs 90207 determined by the British Hypertension Society protocol. J Hypertens 1991, 9:573-574.

20. Gill JS, Zezulka AV, Beevers DG, Davies P: Relation between initial blood pressure and its fall with treatment. Lancet 1985, 1:567-569.

21. Snedecor GW, Cochran WG: Statistical methods. 7 edition. Ames IA,: Iowa State Univ. Press; 1980 
22. Risch N, Merikangas K: The future of genetic studies of complex human diseases. Science 1996, 273:1516-1517.

23. Cannella G, Paoletti E, Barocci S, Massarino F, Delfino R, Ravera G, Di Maio G, Nocera A, Patrone P, Rolla D: Angiotensin-converting enzyme gene polymorphism and reversibility of uremic left ventricular hypertrophy following long-term antihypertensive therapy. Kidney Int 1998, 54:618-626.

24. Levy D, Ehret GB, Rice K, Verwoert GC, Launer LJ, Dehghan A, Glazer NL, Morrison AC, Johnson AD, Aspelund T, et al: Genome-wide association study of blood pressure and hypertension. Nat Genet 2009, 41:677-687.

25. Bell KJ, Hayen A, Macaskill P, Craig JC, Neal BC, Fox KM, Remme WJ, Asselbergs FW, van Gilst WH, Macmahon S, et al: Monitoring initial response to Angiotensin-converting enzyme inhibitor-based regimens: an individual patient data meta-analysis from randomized, placebocontrolled trials. Hypertension 2010, 56:533-539.

26. Macrae CA, Vasan RS: Next-generation genome-wide association studies: time to focus on phenotype? Circ Cardiovasc Genet 2011, 4:334-336.

27. Mokwe E, Ohmit SE, Nasser SA, Shafi T, Saunders E, Crook E, Dudley A, Flack JM: Determinants of blood pressure response to quinapril in black and white hypertensive patients: the Quinapril Titration Interval Management Evaluation trial. Hypertension 2004, 43:1202-1207.

doi:10.1186/1479-5876-10-47

Cite this article as: Turner et al:: Power to identify a genetic predictor of antihypertensive drug response using different methods to measure blood pressure response. Journal of Translational Medicine 2012 10:47.

\section{Submit your next manuscript to BioMed Central} and take full advantage of:

- Convenient online submission

- Thorough peer review

- No space constraints or color figure charges

- Immediate publication on acceptance

- Inclusion in PubMed, CAS, Scopus and Google Scholar

- Research which is freely available for redistribution

Submit your manuscript at www.biomedcentral.com/submit
Biomed Central 